\title{
Effect of exchange-correlation functionals on the density functional theory simulation of phase transformation of fast-ion conductors: a case study in the Li garnet oxide $\mathrm{Li}_{7} \mathrm{La}_{3} \mathrm{Zr}_{2} \mathrm{O}_{12}$
}

Matthew J. Klenk, Wei Lai*

Department of Chemical Engineering and Materials Science, Michigan State University, East Lansing, Michigan, USA

*laiwei@msu.edu

\begin{abstract}
The phase transformation of a fast-ion conductor $\mathrm{Li}_{7} \mathrm{La}_{3} \mathrm{Zr}_{2} \mathrm{O}_{12}$ (LLZ) upon heating was investigated using first-principle molecular dynamics by applying the local density approximation (LDA) and thirteen generalized gradient approximations (GGA) of the electron exchange and correlation (XC) energy functionals within the densityfunctional theory (DFT) framework. It was found that some functionals in the selected group failed to predict the phase transformation behavior while others predicted too large or small lattice volumes. Of the fourteen, three functional types, PBEsol, SOGGA, and PBE2, exhibited behaviors consistent with the tetragonal to cubic phase transformation upon heating and they were able to reproduce crystallite volumes within $1.5 \%$ of the experimental values. The correlation of XC functional forms and their accuracy in predicting materials properties was discussed.
\end{abstract}

\section{Introduction}


Research focusing on simulating physical and chemical properties of materials has grown tremendously as the raw computational power and algorithm efficiency have improved drastically over the past decades. Density functional theory (DFT) $)^{1,2}$ has resultantly become a common simulation technique to predict the thermodynamic and kinetic properties of a variety of simple and complex materials. Within the DFT framework, the electron exchange-correlation (XC) energy functional is not known $a$ priori and approximate functionals have to be employed. For example, the libXC package contains about 180 functionals and it is expected that more will be developed and added.

The simplest XC functional is the local density approximation (LDA $)^{2}$ which approximates the $\mathrm{XC}$ energy density at a local position by the value of a uniform electron gas (UEG) with the same electron density. The next two rungs on the Jacob's ladder $^{4}$ are generalized gradient approximation ${ }^{5}$ (GGA) which includes the electron density gradient and meta-GGA which further includes the Laplacian of the density. Within each family of approximation, many different functional forms on the density, gradient, or Laplacian have been proposed based on the electron gas with slowlyvarying density, ${ }^{5}$ Airy gas, ${ }^{6}$ or semiclassical neutral atom. ${ }^{7}$ Due to the wide range of structure features and bonding environment within complex materials, choosing the proper functional for a system can be difficult. This is one of the reasons that much effort has been devoted to test a variety of XC functionals for different solids to see 
how simulation results are close to experimental values (lattice constants, bulk modulus, cohesive energy etc), e.g. by Haas et al in $2009^{8,9}$, Csonka et al in $2009^{10}$, Hao et al in $2012^{11}$, Labat et al in $2013^{12}$, He et al in $2014^{13}$, Rasander et al in $2015^{14}$, Tran et al in $2016 .{ }^{15}$ However, most of these studies are focused on the static structure $(0 \mathrm{~K})$ of simple metals and binary compounds.

Fast-ion conductors are complex materials with at least one mobile species and often exhibit a phase transformation from a low-symmetry structure to a highsymmetry structure upon heating. At present, the effect of XC functionals on simulated materials properties of fast-ion conductors remains elusive. The objective of the present work is to evaluate a collection of $X C$ functionals on the phase transformation of a model fast-ion conductor: lithium garnet oxide $\mathrm{Li}_{7} \mathrm{La}_{3} \mathrm{Zr}_{2} \mathrm{O}_{12}$ (LLZ). LLZ was chosen because of its high lithium ionic conductivity, importance to solidstate battery research, and characteristic phase transformation from a tetragonal to a cubic phase around $900 \mathrm{~K} \cdot{ }^{16-18}$ LLZ has been the subject to several DFT studies, e.g. Xu et $\mathrm{al}^{19}$, Bernstein et $\mathrm{al}^{20}$, Jalem et $\mathrm{al}^{21}$, Meier et $\mathrm{al}^{22}$, Rettenwander et $\mathrm{al}^{23}$, Miara et a $\mathrm{l}^{24}$, Kang et $\mathrm{al}^{25}$ but only one XC functional was employed in each of these investigations. In this work, we selected 14 different XC functionals (LDA, 13 GGA including 2 dispersion corrected ones) and recorded the dynamic response of the LLZ system over the course of a simulation to categorize if or what type of transformation occurs. We then compared the average crystal structure at different temperatures to experimental 
results in order to determine the best XC functional for this system. It is expected such investigation could also provide insight into studies of other fast-ion conductors.

\section{Computational details}

The first-principles DFT-based molecular dynamics computation was performed by the Quickstep $\operatorname{code}^{26}$ implemented in the $c p 2 \mathrm{k}^{27}$ package, which employs mixed Gaussian and plane wave (GPW) basis sets. The valence electron configurations were La $\left(5 s^{2} 5 p^{6} 6 s^{2} 5 d^{1}\right), \operatorname{Li}\left(1 s^{2} 2 s^{1}\right), \operatorname{Zr}\left(4 s^{2} 4 p^{6} 5 s^{2} 4 d^{2}\right)$, and $O\left(2 s^{2} 2 p^{4}\right)$ with Godecker-TetterHutter (GTH) norm-conserving pseudo potentials. ${ }^{28,29}$ The plane wave cutoff was 400 Ry (convergence study shown in the supplementary) and the Gaussian basis sets were molecular optimized double zeta-valence basis sets with a polarization function (DZVP). ${ }^{30}$ The simulation cell was a unit cell of $\mathrm{Li}_{7} \mathrm{La}_{3} \mathrm{Zr}_{2} \mathrm{O}_{12}$ with 192 atoms and 1200 valence electrons, sampled at the gamma point. The constant number/pressure/temperature (NPT) simulation was performed for 6 or 12 ps (1 fs time step) at different temperatures and zero pressure, with a canonical sampling through velocity rescaling (CSVR) thermostat ${ }^{31}$ (time constant of $5 \mathrm{fs}$ ) and MartynaTuckerman-Tobias-Klein (MTTK) barostat ${ }^{32}$ (time constant of $300 \mathrm{fs}$ ). Local density approximation (LDA) and thirteen generalized gradient approximation (GGA) XC functionals, implemented in the original and modified LibXC ${ }^{3}$ package were investigated. GGA functionals, except $\mathrm{PW} 91^{33}$ and $\mathrm{AM} 05^{6}$, have 9 different PBE-like functional forms. The dependence of exchange enhancement factor $F_{X}(s)$ of these 9 
functionals on the reduced density gradient, i.e. $s$, is presented in Table 1 . They are mainly composed of two parameters, $\mu$ and $\kappa$. The parameter $\mu$ determines the behavior at small $s$, whose value was set to $\mu_{P B E}$ in the PBE approximation by cancelling second-order exchange and correlation terms. Other values inspired by the gradient expansion of electron gas with slowly-varying density $\left(\mu_{G E}\right)^{34}$ or formationenergy fitting $\left(\mu_{F E}\right)^{8}$ have also been used. On the other hand, parameter $\kappa$ determines the behavior at large $s$, whose value was chosen to be $\kappa_{P B E}$ to satisfy the Lieb-Oxford bound. ${ }^{5}$ Other tightened values inspired by the electron liquid $\left(\kappa_{E L}\right)^{35}$ or formation-energy fitting $\left(\kappa_{F E}\right)$ have been used. ${ }^{8}$ The functional form of correlation ehancement factor $F_{C}(s)$ can be found in the literature. ${ }^{5}$ Modified parameters of $\beta$ in $F_{C}(s)$ and other parameters in $F_{X}(s)$ are also presented in Table 1 . Finally, the dispersion correction in the Grimme D3(BJ) formalism ${ }^{36,37}$ was applied to two of PBElike functionals, PBE and PBEsol.

Table 1: Exchange and correlation enhancement factors of the PBE-like functionals. PBE2 was named as $\operatorname{PBE}\left(G_{c \prime} G_{x \prime} E L\right)$ in the literature. ${ }^{38}$

\begin{tabular}{ccc}
\hline & Exchange $F_{X}(s)$ & $\begin{array}{c}\text { Correlation } \\
F_{C}(s)\end{array}$ \\
\hline $\mathrm{PBE}^{5}$ & $1+\kappa_{P B E}\left(1-\frac{1}{1+\mu_{P B E} s^{2} / \kappa_{P B E}}\right)$ & $\mathrm{PBEC}$ \\
$\mathrm{PBE}^{38}$ & $1+\kappa_{E L}\left(1-\frac{1}{1+\mu_{G E} s^{2} / \kappa_{E L}}\right)$ & $\mathrm{PBEC}$ \\
& $1+\kappa_{P B E}\left(1-\frac{1}{1+\mu_{G E} s^{2} / \kappa_{P B E}}\right)$ & PBEC with \\
$\mathrm{PBEsol}^{34}$ & $1+\kappa_{P B E}\left(1-e^{-\mu_{P B E} s^{2} / \kappa_{P E E}}\right)$ & $\beta=0.046$ \\
$\mathrm{RPBE}^{39}$ & 5 & $\mathrm{PBEC}$
\end{tabular}




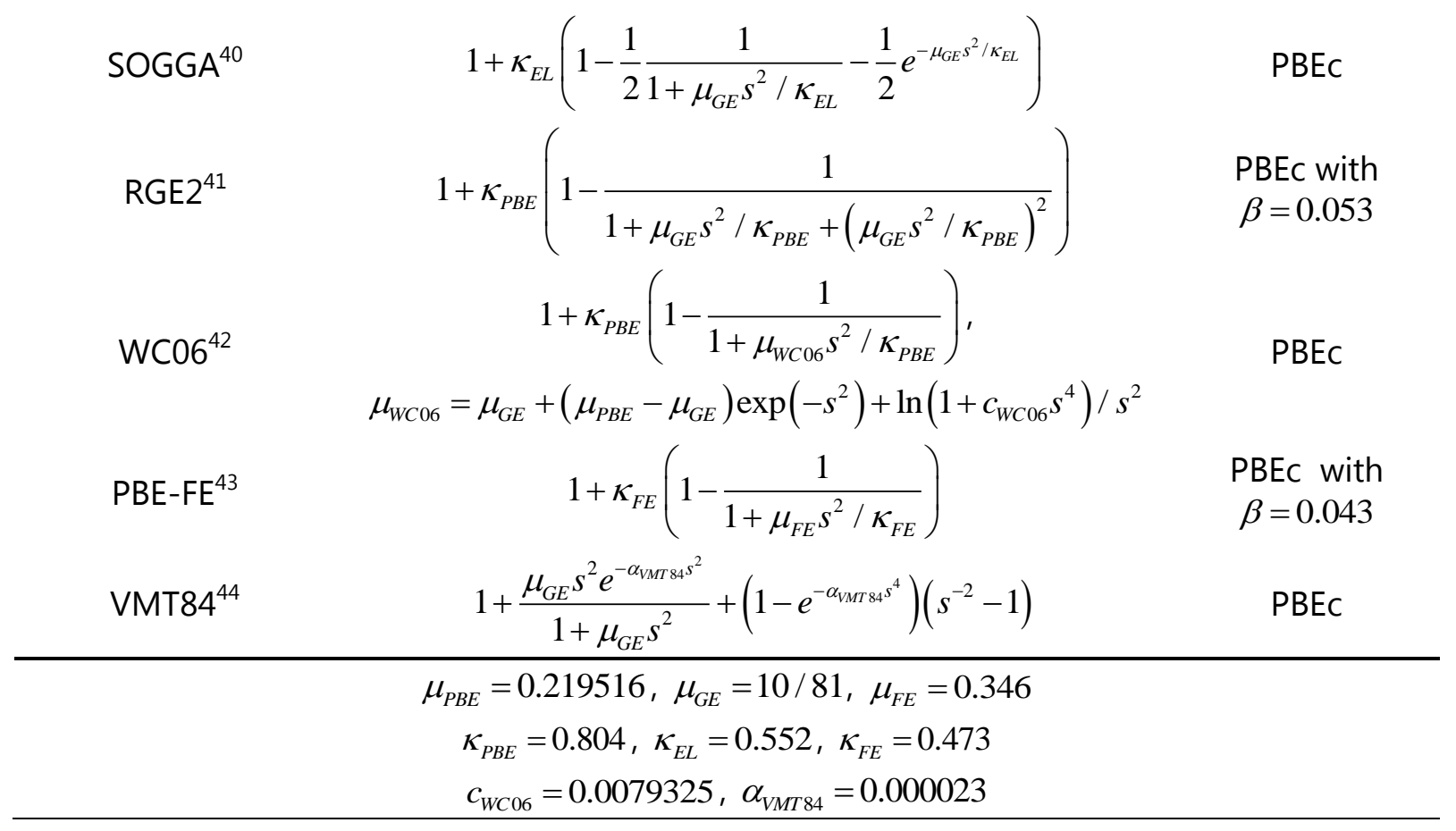

\section{Results and Discussion}

\section{Phase behavior at 1000 K: all 14 functionals}

To first determine whether an XC functional merited further study, an initial structure of tetragonal LLZ based on experimental neutron diffraction data ${ }^{45}$ was heated to $1000 \mathrm{~K}$ to check if a tetragonal to cubic phase transformation could be observed, as the experimental transition temperature is around $900 \mathrm{~K} \cdot{ }^{17,18}$ Figure 1 presents the results of these initial simulations by plotting lattice parameters $(a, b, c)$ as a function of time. In general, lattice variation could be characterized as two categories. First, systems with functionals such as LDA, PBEsol-D3(BJ), VMT84, WC06, PBE-D3(BJ), AM05 and PBE-FE almost always stay as a tetragonal phase in the 


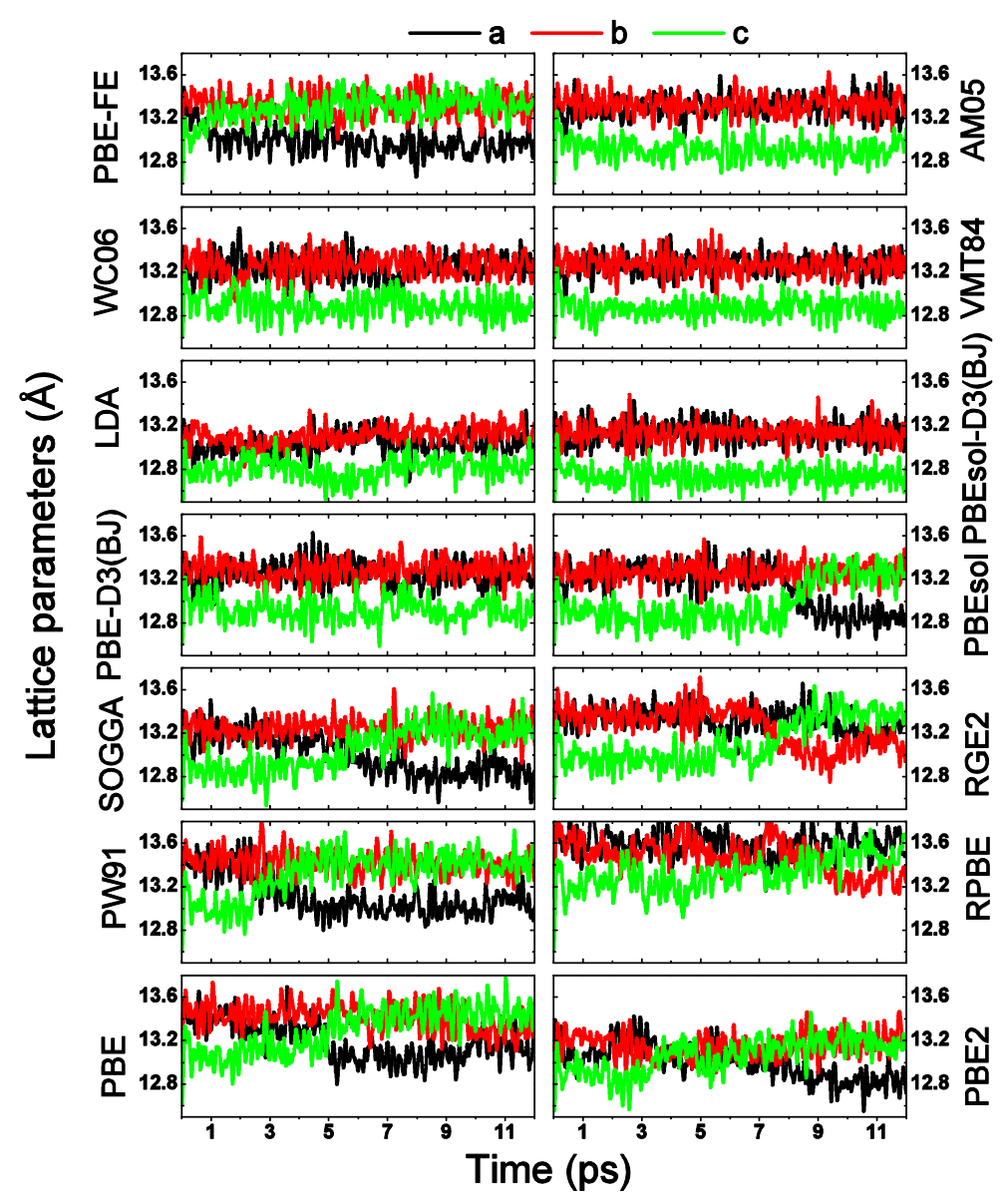

Figure 1: Simulated lattice parameters $(a, b, c)$ as a function of time at $1000 \mathrm{~K}$. PBE-FE, AM05, WC06, VMT84, LDA, PBEsol-D3(BJ), and PBE-D3(BJ) are categorized as tetragonal (T) group, while the rest are categorized as oscillating tetragonal/cubic $\left(T / C^{*}\right)$ group.

duration of simulation. These functionals are categorized as the tetragonal $(\mathrm{T})$ group. The remaining functionals exhibit moments of cubic phase before reverting back to some form of tetragonal structure. They are categorized as the $\mathrm{T} / \mathrm{C}^{*}$ group with varying degree of cubic/tetragonal volatility.

In Table 2, we present the quantitative comparison of lattice volume at $1000 \mathrm{~K}$ obtained from simulation to that of extrapolated X-ray diffraction data from 
Matsuda ${ }^{18}$. We can see that lattice volumes are delimited by LDA (smallest) and RPBE (largest), which can be correlated to how the exchange energy depends on the reduced gradient, to be shown in Figure 3. LDA and PBEsol-D3(BJ) functional underestimate the volume by more than $2 \%$, while PBE, PW91 and RPBE functionals overestimate the volume by more than $3 \%$. Some of the newer functionals specifically designed for solids, e.g. AM05, WC06, SOGGA, PBEsol are able to predict volume values within $1.5 \%$. These two observations were also made in several other studies of effect of XC functionals. ${ }^{8-15}$ Other more recent functionals such as VMT84, PBE-FE, PBE2, and RGE2 have scattered success in obtaining values consistent with experiments. While VMT84 predicts a very accurate lattice volume, it does not predict the right phase behavior. Both PBE-FE and RGE2 slightly overestimate while PBE2 slightly underestimates the volume. When we compare dispersion corrected and noncorrected functionals, it seems that addition of dispersion energy reduces the lattice volume, which brings lattice volume from PBE-D3(BJ) close to experimental values and leads to underestimation from PBEsol-D3(BJ). This observation is similar to those reported in the literature on strongly and weakly bound materials. ${ }^{15}$

Table 2: Summary of simulation results (lattice shape and volume) using different XC functionals.

\begin{tabular}{cccccc}
\hline XC functional & $\begin{array}{c}\text { Lattice } \\
\text { shape }\end{array}$ & \multicolumn{4}{c}{ Lattice volume error (\%) } \\
& $(1000 \mathrm{~K})$ & $1000 \mathrm{~K}$ & $900 \mathrm{~K}$ & $700 \mathrm{~K}$ & $300 \mathrm{~K}$ \\
\hline LDA $^{2}$ & $\mathrm{~T}$ & -2.86 & -3.53 & -3.51 & -3.57 \\
PBEsol-D3(BJ) & $\mathrm{T}$ & -2.56 & & &
\end{tabular}




\begin{tabular}{cccccc} 
VMT84 $^{41}$ & $\mathrm{~T}$ & 0.40 & & & \\
WCO6 $^{36}$ & $\mathrm{~T}$ & 0.46 & & & \\
$\mathrm{PBE}-\mathrm{D} 3(\mathrm{BJ})^{4}$ & $\mathrm{~T}$ & 0.77 & & & \\
$\mathrm{AM05}^{5}$ & $\mathrm{~T}$ & 1.46 & 1.50 & 1.30 & 0.84 \\
$\mathrm{PBE}-\mathrm{FE}^{39}$ & $\mathrm{~T}$ & 1.94 & 1.87 & 1.78 & 1.43 \\
\hline $\mathrm{PBE}^{38}$ & $\mathrm{~T} / \mathrm{C}^{*}$ & -1.02 & -1.25 & -1.00 & -0.01 \\
$\mathrm{SOGGA}^{34}$ & $\mathrm{~T} / \mathrm{C}^{*}$ & -0.36 & -0.43 & -0.45 & -0.72 \\
$\mathrm{PBEsOl}^{42}$ & $\mathrm{~T} / \mathrm{C}^{*}$ & 0.31 & 0.35 & 0.23 & -0.01 \\
$\mathrm{RGE2}^{35}$ & $\mathrm{~T} / \mathrm{C}^{*}$ & 2.70 & & & \\
$\mathrm{PW}^{31}$ & $\mathrm{~T} / \mathrm{C}^{*}$ & 3.87 & 3.81 & 3.49 & 2.97 \\
$\mathrm{PBE}^{4}$ & $\mathrm{~T} / \mathrm{C}^{*}$ & 4.30 & 4.12 & 3.87 & 3.35 \\
$\mathrm{RPBE}^{33}$ & $\mathrm{~T} / \mathrm{C}^{*}$ & 8.17 & & & \\
\hline
\end{tabular}

\section{Lattice parameters at all temperatures: 8 functionals}

Based on results in Figure 1/Table 2 and popularity of functionals in the field, we further selected 8 functionals, i.e. AM05, LDA, PW91, PBE-FE, SOGGA, PBEsol, PBE, and PBE2, to perform simulation at lower temperatures $(900,700$, and $300 \mathrm{~K})$ to investigate the phase transformation and lattice volumes. Plots of the time dependent lattice parameters at $900 \mathrm{~K}, 700 \mathrm{~K}$, and $300 \mathrm{~K}$, similar to Figure 1, can be found in the supplemental material.

Figure 2 consists of the time averaged lattice parameters with standard deviation for LLZ at all 4 simulated temperatures. Each simulation is then compared to the X-ray diffraction structure reported by Matsuda et al. ${ }^{18}$ Their study shows a transformation temperature of about $927 \mathrm{~K}$, consistent with our experimental investigation of impurity free LLZ. ${ }^{17}$ While all structures in Figure 2 maintain consistent 
tetragonal character below $900 \mathrm{~K}$, we consider that four functionals, i.e. SOGGA,

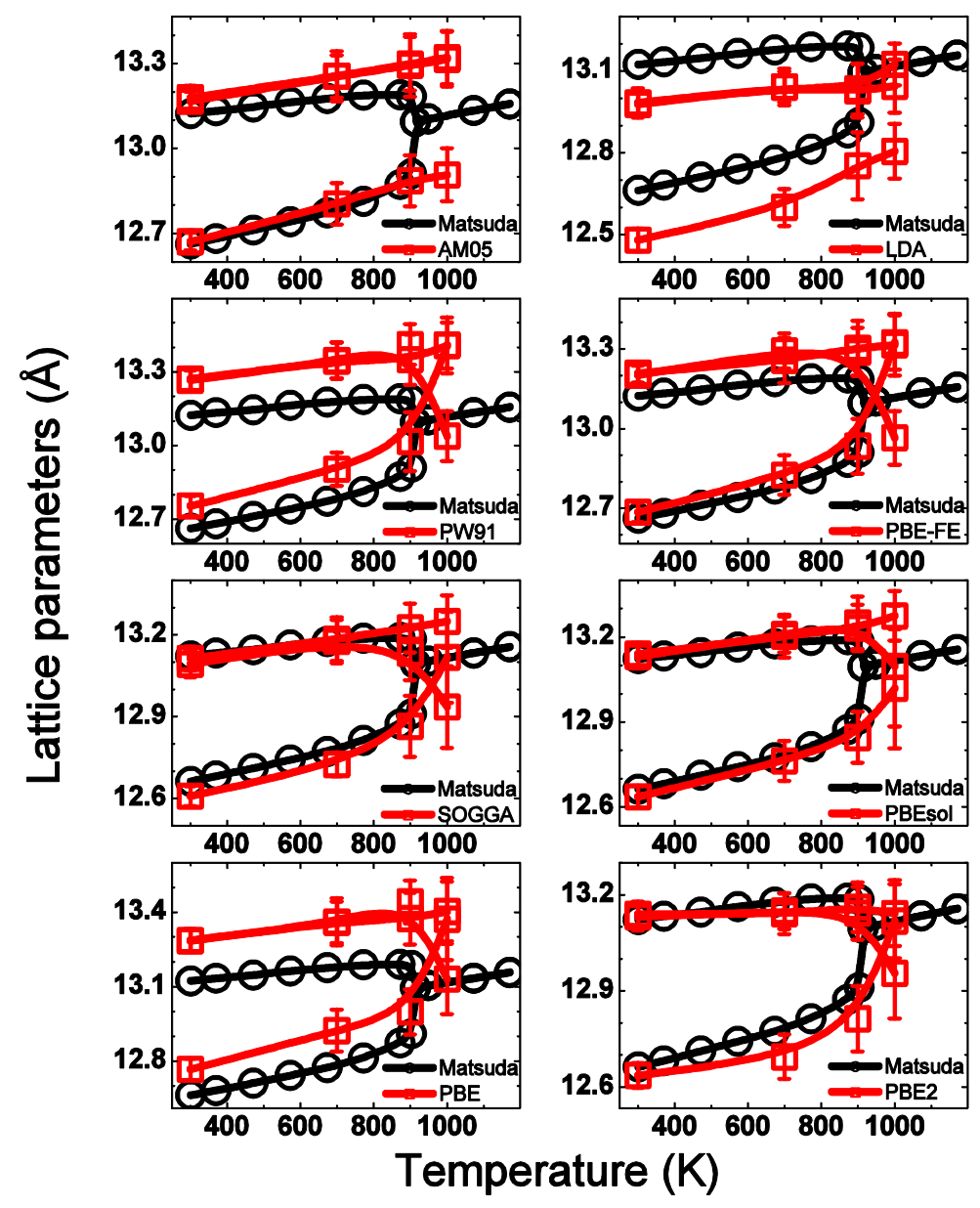

Figure 2: Average lattice parameters for the eight $X C$ functionals at different temperatures compared to XRD extracted lattice parameters by Matsuda.

PBEsol, PBE, and PBE2 functionals are able to predict a cubic-like average structure at $1000 \mathrm{~K}$ due to the overlapping standard deviations. Out of these four, SOGGA, PBEsol, and PBE2 are able to predict lattice volume within $1.5 \%$ of experimental values at all temperatures.

\section{Correlation between XC functionals and phase behavior/volume}


It is common in the literature to plot the density gradient dependence of the exchange 
enhancement factor to examine the difference of $X C$ functionals, since there is generally less variation in the correlation energy. The plot of different GGA functionals employed in this study is shown in Figure 3. The second-order gradient expansion (GE) of electron gas with slowly-varying density is shown as the dotted line. In general, it is proposed that a functional will overestimate the volume if its enhancement factor is larger than the gradient expansion at small $s$. Indeed this is the case for RPBE, PBE, PW91, and PBEFE. On the other hand, functionals with similar values to the dotted line at small $s$ generally can predict volumes close to experiments, e.g. PBEsol, WC06, SOGGA, PBE2, with the exception of RGE2 and VMT84, both of which have higher values than the other four at large $s$. This suggest that functional forms at both small and large $s$ range affect the predicted lattice volumes.

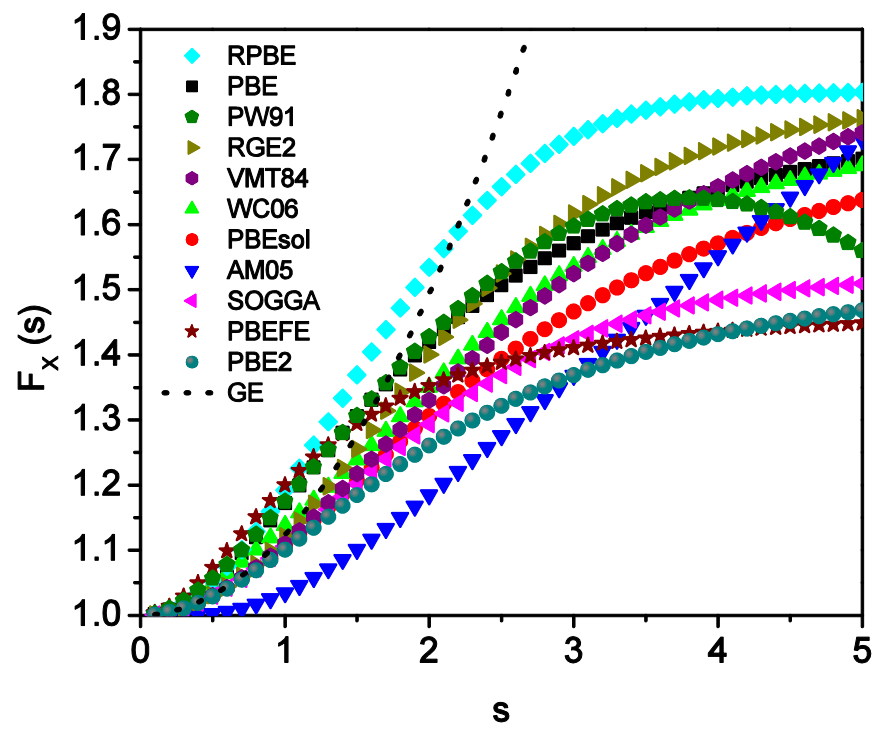

Figure 3: Plot of exchange enhancement factors of GGA functionals employed in the study. The second order gradient expansion as $1+\mu_{G E} s^{2}$ is shown in the dotted line. 
This is also illustrated with the AM05 functional whose deviation from the GE line at small $s$ is likely to be compensated by its large value at large $s$. Out of the four functionals with good accuracy in lattice volume, PBEsol, SOGGA, PBE2, and WC06, the first three are also able to predict the right phase behavior, possibly due to their lower values at large $s$ compared with WC06.

We think part of the difficulty of these GGA functionals to predict correctly both the phase transformation behavior and lattice volume is the complex bonding environment (a mix of ionic and covalent bonding with different elements) in $\mathrm{Li}_{7} \mathrm{La}_{3} \mathrm{Zr}_{2} \mathrm{O}_{12}$, along with the intrinsic limitation of GGA approximations as they only depend on the gradient ( $1^{\text {st }}$ order) of density. It was proposed that approximations involving higher-order terms (Laplacian of density), i.e. Meta-GGA, could lead to more accurate results within the DFT framework, as demonstrated with the Minnesota MetaGGA MN15- ${ }^{46}$ and Strongly Constrained and Appropriately Normed (SCAN) functionals ${ }^{47}$. We plan to investigate the effect of these Meta-GGA functionals in the future.

\section{Conclusion}

The present study shows that molecular dynamics modeling of phase transformation of fast-ion conductors within the DFT framework can be a challenging task as it strongly depends on the employed XC functional. For the PBE-like GGA functional, the 
functional forms at both small and large density gradient ranges affects whether the correct lattice volume or phase behavior can be obtained. Of the fourteen functionals studied, SOGGA, PBEsol, and PBE2 show strong agreement with experimental crystal structures of LLZ and appear to be candidates for further study into the dynamics of lithium diffusion in this material family.

\section{Acknowledgements}

We wish to acknowledge the computing resources provided by the Michigan State University High Performance Computing Center and the Extreme Science and Engineering Discovery Environment (XSEDE), which is supported by National Science Foundation grant number ACI-1053575. We acknowledge the financial support by the Ceramics Program of National Science Foundation (DMR-1206356).

\section{References}

(1) P. Hohenberg, and W. Kohn, "INHOMOGENEOUS ELECTRON GAS," Physical Review B, 136 (1964) B864-+. http://dx.doi.org/10.1103/PhysRev.136.B864

(2) W. Kohn, and L. J. Sham, "SELF-CONSISTENT EQUATIONS INCLUDING EXCHANGE AND CORRELATION EFFECTS," Physical Review, 140 (1965) 1133-\&. http://dx.doi.org/10.1103/PhysRev.140.A1133

(3) M. A. L. Marques, M. J. T. Oliveira, and T. Burnus, "LIBXC: A library of exchange and correlation functionals for density functional theory," Comput. Phys. Commun., 183 (2012) 2272-2281. http://dx.doi.org/10.1016/j.cpc.2012.05.007

(4) J. P. Perdew, and K. Schmidt, In Density Functional Theory and Its Application to Materials; VanDoren, V., VanAlsenoy, C., Geerlings, P., Eds. 2001; Vol. 5771-20.

(5) J. P. Perdew, K. Burke, and M. Ernzerhof, "Generalized gradient approximation made simple," Phys. Rev. Lett., 77 (1996) 3865-3868. http://dx.doi.org/10.1103/PhysRevLett.77.3865 
(6) A. E. Mattsson, R. Armiento, J. Paier, G. Kresse, J. M. Wills, and T. R. Mattsson, "The AM05 density functional applied to solids," J. Chem. Phys., 128 (2008).

http://dx.doi.org/10.1063/1.2835596

(7) L. A. Constantin, A. Terentjevs, F. Della Sala, P. Cortona, and E. Fabiano, "Semiclassical atom theory applied to solid-state physics," Phys. Rev. B, 93 (2016).

http://dx.doi.org/10.1103/PhysRevB.93.045126

(8) P. Haas, F. Tran, and P. Blaha, "Calculation of the lattice constant of solids with semilocal functionals," Physical Review B, 79 (2009).

http://dx.doi.org/10.1103/PhysRevB.79.085104

(9) P. Haas, F. Tran, and P. Blaha, "Calculation of the lattice constant of solids with semilocal functionals (vol 79, 085104, 2009)," Phys. Rev. B, 79 (2009).

http://dx.doi.org/10.1103/PhysRevB.79.209902

(10) G. I. Csonka, J. P. Perdew, A. Ruzsinszky, P. H. T. Philipsen, S. Lebegue, J. Paier, O. A. Vydrov, and J. G. Angyan, "Assessing the performance of recent density functionals for bulk solids," Phys. Rev. B, 79 (2009). http://dx.doi.org/10.1103/PhysRevB.79.155107

(11) P. Hao, Y. Fang, J. W. Sun, G. I. Csonka, P. H. T. Philipsen, and J. P. Perdew, "Lattice constants from semilocal density functionals with zero-point phonon correction," Phys. Rev. B, 85 (2012). http://dx.doi.org/10.1103/PhysRevB.85.014111

(12) F. Labat, E. Bremond, P. Cortona, and C. Adamo, "Assessing modern GGA functionals for solids," Journal of Molecular Modeling, 19 (2013) 2791-2796.

http://dx.doi.org/10.1007/s00894-012-1646-2

(13) L. H. He, F. Liu, G. Hautier, M. J. T. Oliveira, M. A. L. Marques, F. D. Vila, J. J. Rehr, G. M. Rignanese, and A. H. Zhou, "Accuracy of generalized gradient approximation functionals for density-functional perturbation theory calculations," Phys. Rev. B, 89 (2014).

http://dx.doi.org/10.1103/PhysRevB.89.064305

(14) M. Rasander, and M. A. Moram, "On the accuracy of commonly used density functional approximations in determining the elastic constants of insulators and semiconductors," J.

Chem. Phys., 143 (2015). http://dx.doi.org/10.1063/1.4932334

(15) F. Tran, J. Stelzl, and P. Blaha, "Rungs 1 to 4 of DFT Jacob's ladder: Extensive test on the lattice constant, bulk modulus, and cohesive energy of solids," Journal of Chemical Physics, 144 (2016). http://dx.doi.org/10.1063/1.4948636

(16) R. Murugan, V. Thangadurai, and W. Weppner, "Fast lithium ion conduction in garnettype Li7La3Zr2O12," Angewandte Chemie-International Edition, 46 (2007) 7778-7781.

http://dx.doi.org/10.1002/anie.200701144

(17) Y. X. Wang, and W. Lai, "Phase transition in lithium garnet oxide ionic conductors Li7La3Zr2O12: The role of Ta substitution and H2O/CO2 exposure," Journal of Power Sources, 275 (2015) 612-620. http://dx.doi.org/10.1016/j.jpowsour.2014.11.062

(18) Y. Matsuda, K. Sakamoto, M. Matsui, O. Yamamoto, Y. Takeda, and N. Imanishi, "Phase formation of a garnet-type lithium-ion conductor Li-7 (-) 3xAlxLa3Zr2O12," Solid State lonics, 277 (2015) 23-29. http://dx.doi.org/10.1016/j.ssi.2015.04.011

(19) M. Xu, M. S. Park, J. M. Lee, T. Y. Kim, Y. S. Park, and E. Ma, "Mechanisms of Li+ transport in garnet-type cubic Li3+xLa3M2O12 (M = Te, Nb, Zr)," Phys. Rev. B, 85 (2012) 052301. http://dx.doi.org/10.1103/Physrevb.85.052301 
(20) N. Bernstein, M. D. Johannes, and H. Khang, "Origin of the Structural Phase Transition in Li7La3Zr2O12," Phys. Rev. Lett., 109 (2012) 205702.

http://dx.doi.org/10.1103/PhysRevLett.109.205702

(21) R. Jalem, Y. Yamamoto, H. Shiiba, M. Nakayama, H. Munakata, T. Kasuga, and K. Kanamura, "Concerted Migration Mechanism in the Li Ion Dynamics of Garnet-Type Li7La3Zr2O12," Chem. Mater., 25 (2013) 425-430. http://dx.doi.org/10.1021/Cm303542x

(22) K. Meier, T. Laino, and A. Curioni, "Solid-State Electrolytes: Revealing the Mechanisms of Li-Ion Conduction in Tetragonal and Cubic LLZO by First-Principles Calculations," J. Phys. Chem. C, 118 (2014) 6668-6679. http://dx.doi.org/10.1021/jp5002463

(23) D. Rettenwander, P. Blaha, R. Laskowski, K. Schwarz, P. Bottke, M. Wilkening, C. A. Geiger, and G. Amthauer, "DFT Study of the Role of Al3+ in the Fast Ion-Conductor Li73xAlx3+La3Zr2O12 Garnet," Chem. Mater., 26 (2014) 2617-2623.

http://dx.doi.org/10.1021/cm5000999

(24) L. J. Miara, S. P. Ong, Y. F. Mo, W. D. Richards, Y. Park, J. M. Lee, H. S. Lee, and G. Ceder, "Effect of Rb and Ta Doping on the Ionic Conductivity and Stability of the Garnet Li7+2x$\mathrm{y}($ La3-xRbx)(Zr2-yTay)O-12 $(0<=\mathrm{x}<=0.375,0<=\mathrm{y}<=1)$ Superionic Conductor: A First Principles Investigation," Chem. Mater., 25 (2013) 3048-3055.

http://dx.doi.org/10.1021/Cm401232r

(25) S. G. Kang, and D. S. Sholl, "First-Principles Study of Chemical Stability of the Lithium Oxide Garnets Li7La3M2O12 (M = Zr, Sn, or Hf)," J. Phys. Chem. C, 118 (2014) 17402-17406. http://dx.doi.org/10.1021/jp504314w

(26) J. VandeVondele, M. Krack, F. Mohamed, M. Parrinello, T. Chassaing, and J. Hutter, "QUICKSTEP: Fast and accurate density functional calculations using a mixed Gaussian and plane waves approach," Comput. Phys. Commun., 167 (2005) 103-128.

http://dx.doi.org/10.1016/j.cpc.2004.12.014

(27) J. Hutter, M. Iannuzzi, F. Schiffmann, and J. VandeVondele, "CP2K: atomistic simulations of condensed matter systems," Wiley Interdisciplinary Reviews-Computational Molecular Science, 4 (2014) 15-25. http://dx.doi.org/10.1002/wcms.1159

(28) S. Goedecker, M. Teter, and J. Hutter, "Separable dual-space Gaussian pseudopotentials," Phys. Rev. B, 54 (1996) 1703-1710.

http://dx.doi.org/10.1103/PhysRevB.54.1703

(29) M. Krack, "Pseudopotentials for $\mathrm{H}$ to Kr optimized for gradient-corrected exchangecorrelation functionals," Theor. Chem. Acc., 114 (2005) 145-152.

http://dx.doi.org/10.1007/s00214-005-0655-y

(30) J. VandeVondele, and J. Hutter, "Gaussian basis sets for accurate calculations on molecular systems in gas and condensed phases," J. Chem. Phys., 127 (2007).

http://dx.doi.org/10.1063/1.2770708

(31) G. Bussi, D. Donadio, and M. Parrinello, "Canonical sampling through velocity rescaling," J. Chem. Phys., 126 (2007). http://dx.doi.org/10.1063/1.2408420

(32) G. J. Martyna, M. E. Tuckerman, D. J. Tobias, and M. L. Klein, "Explicit reversible integrators for extended systems dynamics," Mol. Phys., 87 (1996) 1117-1157.

http://dx.doi.org/10.1080/00268979650027054

(33) J. P. Perdew, J. A. Chevary, S. H. Vosko, K. A. Jackson, M. R. Pederson, D. J. Singh, and C. Fiolhais, "ATOMS, MOLECULES, SOLIDS, AND SURFACES - APPLICATIONS OF THE 
GENERALIZED GRADIENT APPROXIMATION FOR EXCHANGE AND CORRELATION," Physical Review B, 46 (1992) 6671-6687. http://dx.doi.org/10.1103/PhysRevB.46.6671

(34) J. P. Perdew, A. Ruzsinszky, G. I. Csonka, O. A. Vydrov, G. E. Scuseria, L. A. Constantin, X. L. Zhou, and K. Burke, "Restoring the density-gradient expansion for exchange in solids and surfaces," Phys. Rev. Lett., 100 (2008). http://dx.doi.org/10.1103/PhysRevLett.100.136406

(35) M. M. Odashima, and K. Capelle, "How tight is the Lieb-Oxford bound?," J. Chem. Phys., 127 (2007). http://dx.doi.org/10.1063/1.2759202

(36) S. Grimme, J. Antony, S. Ehrlich, and H. Krieg, "A consistent and accurate ab initio parametrization of density functional dispersion correction (DFT-D) for the 94 elements H-Pu," J. Chem. Phys., 132 (2010). http://dx.doi.org/10.1063/1.3382344

(37) S. Grimme, S. Ehrlich, and L. Goerigk, "Effect of the Damping Function in Dispersion Corrected Density Functional Theory," J. Comput. Chem., 32 (2011) 1456-1465. http://dx.doi.org/10.1002/jcc.21759

(38) P. Haas, F. Tran, P. Blaha, L. S. Pedroza, A. J. R. da Silva, M. M. Odashima, and K. Capelle, "Systematic investigation of a family of gradient-dependent functionals for solids," Phys. Rev. B, 81 (2010). http://dx.doi.org/10.1103/PhysRevB.81.125136

(39) B. Hammer, L. B. Hansen, and J. K. Norskov, "Improved adsorption energetics within density-functional theory using revised Perdew-Burke-Ernzerhof functionals," Phys. Rev. B, 59 (1999) 7413-7421. http://dx.doi.org/10.1103/PhysRevB.59.7413

(40) Y. Zhao, and D. G. Truhlar, "Construction of a generalized gradient approximation by restoring the density-gradient expansion and enforcing a tight Lieb-Oxford bound," $J$. Chem. Phys., 128 (2008). http://dx.doi.org/10.1063/1.2912068

(41) A. Ruzsinszky, G. I. Csonka, and G. E. Scuseria, "Regularized Gradient Expansion for Atoms, Molecules, and Solids," J. Chem. Theory Comput., 5 (2009) 763-769. http://dx.doi.org/10.1021/ct8005369

(42) Z. G. Wu, and R. E. Cohen, "More accurate generalized gradient approximation for solids," Phys. Rev. B, 73 (2006). http://dx.doi.org/10.1103/PhysRevB.73.235116

(43) R. Sarmiento-Perez, S. Botti, and M. A. L. Marques, "Optimized Exchange and Correlation Semilocal Functional for the Calculation of Energies of Formation," J. Chem. Theory Comput., 11 (2015) 3844-3850. http://dx.doi.org/10.1021/acs.jctc.5b00529

(44) A. Vela, J. C. Pacheco-Kato, J. L. Gazquez, J. M. del Campo, and S. B. Trickey, "Improved constraint satisfaction in a simple generalized gradient approximation exchange functional," Journal of Chemical Physics, 136 (2012). http://dx.doi.org/10.1063/1.3701132

(45) Y. X. Wang, A. Huq, and W. Lai, "Insight into lithium distribution in lithium-stuffed garnet oxides through neutron diffraction and atomistic simulation: Li7-xLa3Zr2-xTaxO12 (x=0-2) series," Solid State Ionics, 255 (2014) 39-49. http://dx.doi.org/10.1016/j.ssi.2013.11.017 (46) H. S. Yu, X. He, and D. G. Truhlar, "MN15-L: A New Local Exchange-Correlation Functional for Kohn-Sham Density Functional Theory with Broad Accuracy for Atoms, Molecules, and Solids," J. Chem. Theory Comput., 12 (2016) 1280-1293.

http://dx.doi.org/10.1021/acs.jctc.5b01082

(47) J. W. Sun, R. C. Remsing, Y. B. Zhang, Z. R. Sun, A. Ruzsinszky, H. W. Peng, Z. H. Yang, A. Paul, U. Waghmare, X. F. Wu, M. L. Klein, and J. P. Perdew, "Accurate first-principles structures and energies of diversely bonded systems from an efficient density functional," Nature Chem., 8 (2016) 831-836. http://dx.doi.org/10.1038/nchem.2535 